\title{
A new architecture for a single-chip multi-channel beamformer based on a standard FPGA
}

Tomov, Borislav Gueorguiev; Jensen, Jørgen Arendt

Published in:

Proceedings of IEEE Ultrasonics Symposium

Link to article, DOI:

10.1109/ULTSYM.2001.992011

Publication date:

2001

Document Version

Publisher's PDF, also known as Version of record

Link back to DTU Orbit

Citation (APA):

Tomov, B. G., \& Jensen, J. A. (2001). A new architecture for a single-chip multi-channel beamformer based on a standard FPGA. In Proceedings of IEEE Ultrasonics Symposium (Vol. 2, pp. 1529-1533). IEEE.

https://doi.org/10.1109/ULTSYM.2001.992011

\section{General rights}

Copyright and moral rights for the publications made accessible in the public portal are retained by the authors and/or other copyright owners and it is a condition of accessing publications that users recognise and abide by the legal requirements associated with these rights.

- Users may download and print one copy of any publication from the public portal for the purpose of private study or research.

- You may not further distribute the material or use it for any profit-making activity or commercial gain

- You may freely distribute the URL identifying the publication in the public portal 


\title{
A new architecture for a single-chip multi-channel beamformer based on a standard FPGA
}

\author{
Borislav Gueorguiev Tomov* and Jørgen Arendt Jensen \\ Center for Fast Ultrasound Imaging, Ørsted•DTU, Build. 348, \\ Technical University of Denmark, DK-2800 Kgs. Lyngby, Denmark
}

\begin{abstract}
A new architecture for a compact medical ultrasound beamformer has been developed. Combination of novel and known principles has been utilized, leading to low processing power requirements and simple analog circuitry. Usage of a field programmable gate array (FPGA) for the digital signal processing provides programming flexibility.

First, sparse sample processing is performed by generating the in-phase and quadrature beamformed signals. Hereby only 512 samples are beamformed for each line in an image. That leads to a 15 -fold decrease in the number of operations and enables the use of Delta-Sigma $(\Delta \Sigma)$ modulation analog-to-digital converters (ADC).

Second, simple second-order $\Delta \Sigma$ modulation ADC with classic topology is used. This allows for simple analog circuitry and a very compact design. Several tens of these together with the corresponding preamplifiers can be fitted together onto a single analog integrated circuit.

Third, parameter driven delay generation is used, using 3 input parameters per line per channel for either linear array imaging or phased array imaging. The delays are generated on the fly. The delay generation logic also determines the digital apodization by using 2 additional parameters. The control logic consists of few adders and counters and requires very limited resources.

Fourth, the beamformer is fully programmable. Any channel can be set to use an arbitrary delay curve, and any number of these channels can be used together in an extendable modular multi-channel system.

A prototype of the digital logic is implemented using a Xilinx Virtex-E series FPGA. A $5 \mathrm{MHz}$ center frequency is used along with an oversampling ratio of 14. The sampling clock frequency used is $140 \mathrm{MHz}$ and the number of channels in a single Xilinx 1 million gate FPGA XCV600E is 32. The beamformer utilizes all of the BlockRAM of the device and $33 \%$ of
\end{abstract}

*E-mail: bt@oersted.dtu.dk its Core Logic Block (CLB) resources.

Both simulation results and processed echo data form a phantom are presented.

\section{Introduction}

Making sophisticated technology more accessible is an important consideration in system design. Since digital electronics is rapidly evolving, moving processing functions from analog to digital electronics is a powerful approach which allows for increased flexibility and compactness of the mixedsignal devices.

$\Delta \Sigma$ modulation (DSM) [1] is one of the techniques that make it possible to decrease the complexity of the analog interface electronics by using digital logic. A DSM ADC consists of consecutive stages containing low-pass filters and decimators. The reconstructed samples represent the input signal at equidistant time instances. Ultrasound beamformers though require non-regular sampling because of the delay profiles in receive. A number of researchers have tried to incorporate DSM ADCs into ultrasound beamformers. Freeman et al. [2] have developed a modified modulator architecture in order to facilitate the delay profiles in the beamforming without interrupting the modulation process. Since the oversampling ratio (OSR) is crucial for the amplitude resolution of a DSM ADC, the same research group suggested base-band demodulation [3]. Kozak and Karaman [4] have proposed a beamformer featuring DSM with a non-uniform sampling clock.

In the present paper, several novel techniques are combined in a new beamformer architecture. First, sparse sample processing is employed, leading to about 15 -fold decrease in the necessary operations as only 512 samples per image line are processed. The samples are chosen at the precise time instances, discretized by the sampling frequency of the DSM. Second, each channel uses a circular buffer at the output of 


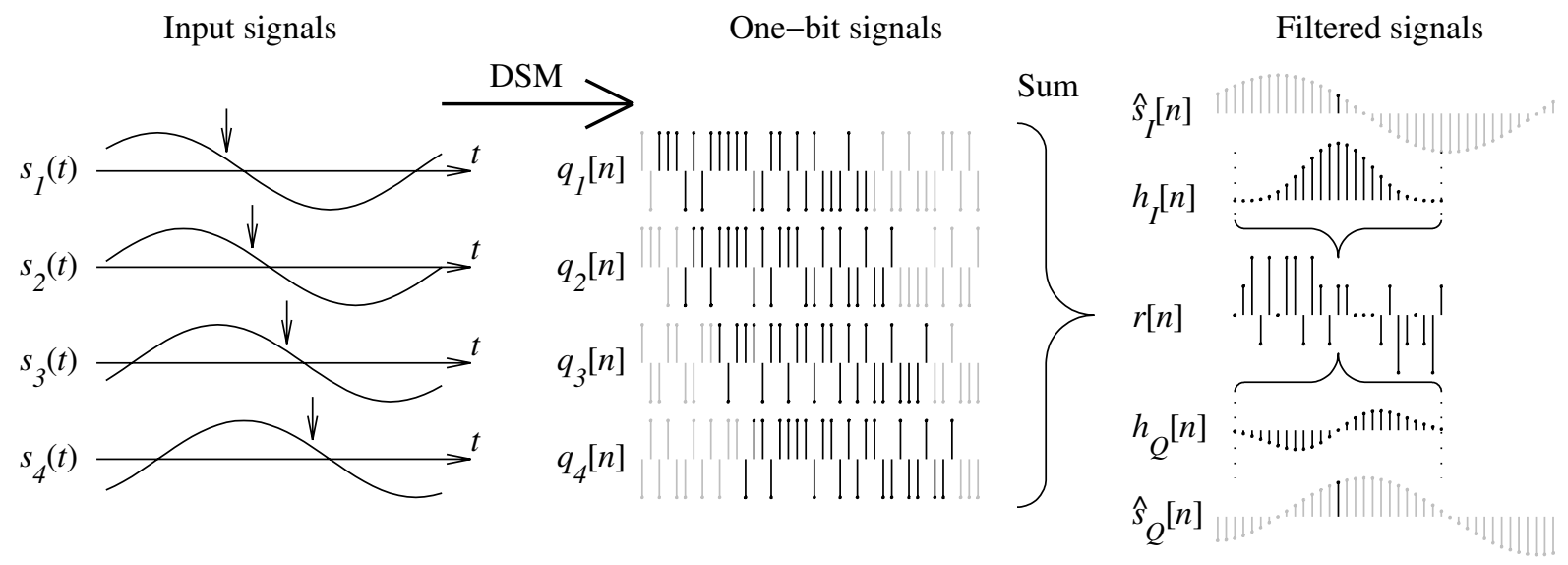

Figure 1: Signal processing of the proposed beamformer, illustrated with 4 channels

the DSM for extraction of the necessary data. Thus, the structure does not impose any restrictions or requirements on the order or topology of the DSM, allowing for flexibility and interchangeability of the analog front-end. Third, the delay generation is parametric and allows independent on-the-fly delay generation for each channel. The calculation scheme is inspired by the Bresenham drawing algorithm [5].

\section{Principles behind the suggested beamformer architecture}

\subsection{Rationale for the sparse sample processing}

The ultrasound images are displayed on raster devices - CRT or LCD displays, which rarely use a resolution beyond that of a TV (525 lines for NTSC) or VGA (640x480 pixels). Therefore, on such displays a beamformed line in an image is represented by no more than 512 points. Thus, it is sufficient to have the correct envelope and phase of the RF signal in 512 equidistant points along the beamformed line to present a correct B-mode or color-flow image. A similar approach was proposed in a different context by Karaman et al. [6].

\subsection{Usage of the Delta-Sigma modulator in the beamformer}

The principle of the DSM implies that the appropriately filtered output of a DSM approximates the input signal, and the approximation improves with increasing the oversampling ratio (OSR). If a filter is applied directly on the DSM output stream, valid output samples can be reconstructed at any clock cycle. In this way, the delay resolution in a beamforming process will be equal to the period of the inherently high modulation frequency.

The signal processing is illustrated in Fig. 1. The analog input signals $s_{k}(t)$ ( $k$ is channel index) from different channels are modulated into bit streams $q_{k}[n]$ in the DSM. In order to perform beamforming at a given point indicated by arrows in the plots of $s_{k}(t)$, sequences of bits (shown in black) are extracted from the streams $q_{k}[n]$, at places that correspond to the appropriate channel delays. The length of the sequences is equal to the length of the reconstruction filters that will be used. The selected sequences are summed into sequence $r[n]$. The latter is then weighted by in-phase $h_{I}[n]$ and quadrature $h_{Q}[n]$ filters to yield selected samples of the in-phase $\hat{s}_{I}[n]$ and quadrature $\hat{s}_{Q}[n]$ reconstructed streams. The matched filter from the classic beamforming is used as in-phase filter and its Hilbert transform is used the quadrature filter, since they suppress the quantization noise to a sufficient degree.

\subsection{Delay generation}

A delay calculation scheme is suggested that allows on-thefly delay generation. It approximates the analytic delay curve for a given imaged line and receiver element. A similar approach with different calculation scheme has been suggested by Feldkämper et al. [7] for increasing the delay resolution in beamformers.

The geometry behind the delay calculation algorithm is shown in Fig. 2. The distance to the focus point $\mathrm{P}$ along the scan line is denoted $d$ and the echo path is denoted $d_{r}$. The full path of the ultrasound wave is denoted $p$. The aperture distance between the emission center and the receiving element is denoted with $x$ and the angle between the scan line and the normal to the transducer surface is denoted $\varphi$. 


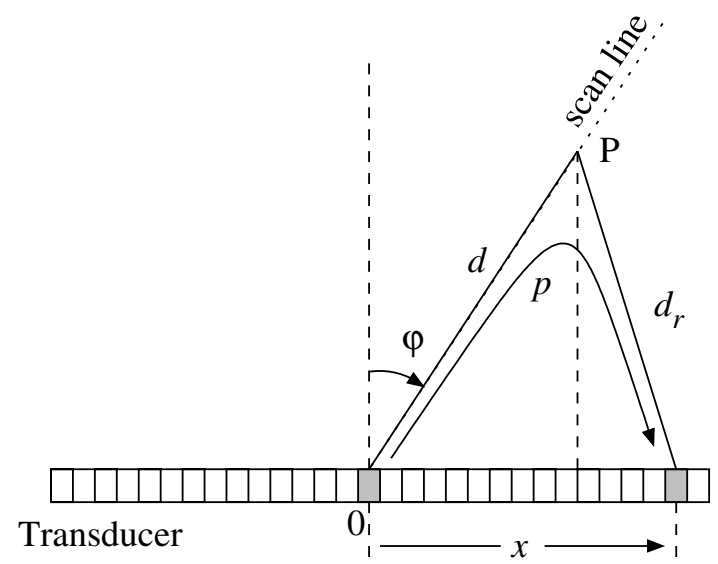

Figure 2: Delay calculation geometry

The echo path $d_{r}$ can be expressed as:

$$
d_{r}=p-d=\sqrt{(x-d \sin \varphi)^{2}+(d \cos \varphi)^{2}} .
$$

After some transformations the equation

$$
p^{2}-2 d(p-x \sin \varphi)-x^{2}=0
$$

is obtained, which describes the imaged line. The term $x \sin \varphi$ is constant for a given line inclination and element and is denoted $k$. For converting the variables into units of clock cycles (for calculations in hardware), both sides of (2) have to be multiplied by $\left(\frac{f_{s}}{c}\right)^{2}$, where $f_{s}$ is the sampling frequency and $c$ is the speed of sound. Eq. (2) becomes:

$$
f\left(p_{N}, d_{N}\right) \equiv p_{N}^{2}-2 d_{N}\left(p_{N}-k_{N}\right)-x_{N}^{2}=0,
$$

where the index $N$ denotes that the variable unit is clock cycle.

In order to keep the focus on the imaged line, the delay generation logic has to keep $f\left(p_{N}, d_{N}\right)$ as close to 0 as possible, therefore it should increase $p_{N}$ by 1 or 2 for each unit increase of $d_{N}$. The choice ${ }^{1}$ is made by evaluating the sign of the function $f\left(p_{N}+1, d_{N}+1\right)$. It can be seen that:

$f\left(p_{N}+1, d_{N}+1\right)=f\left(p_{N}, d_{N}\right)-2 d_{N}+2 k_{N}-1<f\left(p_{N}, d_{N}\right)$

and

$f\left(p_{N}+2, d_{N}+1\right)=f\left(p_{N}, d_{N}\right)+2 p_{N}-4 d_{N}+2 k_{N}>f\left(p_{N}, d_{N}\right)$.

Therefore the following algorithm is suggested:

1. The initial values $d_{N}(1)=d_{\text {start }} \frac{f_{s}}{c}, p_{N}(1)=p_{\text {start }} \frac{f_{s}}{c}$, and $k_{N}=x \sin \varphi \frac{f_{s}}{c}$ are supplied.

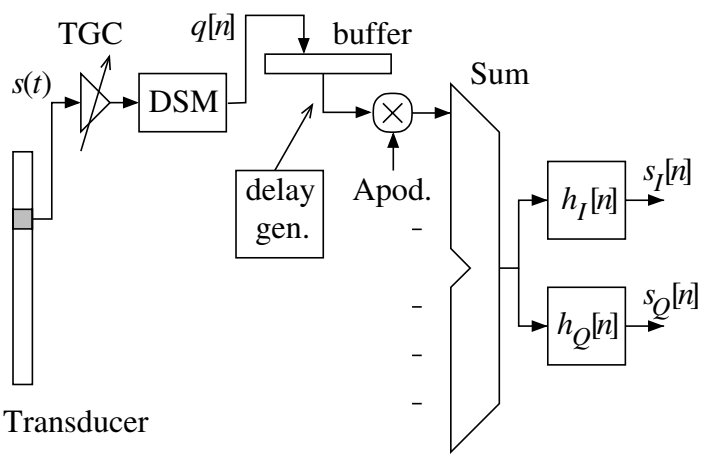

Figure 3: Beamformer architecture

2. If $f\left(p_{N}+1, d_{N}+1\right)>0$, then $p_{N}(n+1)=p_{N}(n)+1$, else $p_{N}(n+1)=p_{N}(n)+2$.

3. If the end of the line is not reached, go to 2 .

The described algorithm approximates the analytical dynamic delay curve within \pm 1 clock cycle.

\section{Beamformer architecture}

The suggested beamformer architecture is shown in Fig. 3. The received RF signal $s(t)$ from every active transducer element is amplified by a variable-gain amplifier (used for timegain compensation) and is converted into high-frequency 1bit digital signal $q[n]$ in the DSM. That data stream is written to a circular buffer. At time instances determined by the delay generation logic, sequences from the stream are read. After multiplication by the apodization coefficient (the weight) of that channel, the aligned sequences corresponding to a given line point are summed across all channels. The result is then filtered for extracting the in-phase and the quadrature components $\hat{s}_{I}[n]$ and $\hat{s}_{Q}[n]$.

\section{Implementation tradeoffs and choices}

The described architecture was implemented in hardware with the following beamformer parameters:

$\begin{array}{lr}\text { Parameter } & \text { Value } \\ \text { Speed of sound } & 1540 \frac{\mathrm{m}}{\mathrm{s}} \\ \text { Center frequency } f_{0} & 5 \mathrm{MHz} \\ \text { Excitation } & 2 \text { sinusoids at } f_{0} \\ \text { Oversampling ratio (OSR) } & 14 \\ \text { Number of channels } & 32\end{array}$

\footnotetext{
${ }^{1}$ In case of tilted line, $p_{N}$ could also stay unchanged at some increase of $d_{N}$. The decision requires evaluation of the sign of $f\left(p_{N}, d_{N}+1\right)$.
} 


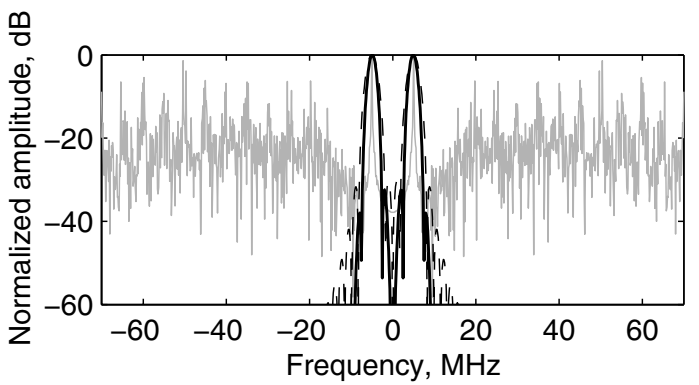

Figure 4: Frequency response of perfect matched filter (solid line) a shortened one (dashed line), along with typical DSM output spectrum (gray).

An important design decision is the choice of the in-phase and the quadrature filters. The matched filter from classic beamforming ( time reversed excitation convolved twice with the impulse response of the transducer) provides excellent suppression of the quantization noise. The length of the filters though is constrained by the amount of clock cycles that are available for producing a reconstructed sample. That number is inversely proportional to the density of the beamformed points. For instance, if 512 points should represent a depth range of $0.15 \mathrm{~m}$, there are between 26 and 53 clock cycles available to the filter block for producing in-phase and the quadrature reconstructed samples.

In the current design, the filtering operation is parallelized in four, so in-phase and quadrature filters with length up to 104 can be used. A perfect matched filter for the simulation setup has length of 168 . Therefore, a number of pseudomatched filters were investigated. The frequency response of the perfect matched filter and that of a shortened one ( 3 central frequency sinusoids, Hamming window weighted) is shown in Fig. 4.

The point spread functions (PSF) of single scaterers in the transmit focal point were obtained through simulations using the two mentioned filters. They were compared against the reference beamforming PSF (Fig. 5) .

The implementation target is the Xilinx Virtex-E FPGA device family which features quite a high number of dualported fast SRAM that can be used as buffers for the DSM output stream. A number of specific design choices are made:

1. The output word of the delay buffer is wider than the input one. This speeds up the reading and allows for parallel processing of the data at the highest possible clock rate.

2. Each calculation cycle in the delay generation requires two clock cycles, therefore a more aggressive computational scheme is employed: $d_{N}$ is increased by 2 for each computation cycle and the sign of $f(d+2, p+1)$, $f(d+2, p+2)$ and $f(d+2, p+3)$ determines whether $p_{N}$ should be increased by $1,2,3$, or 4 .
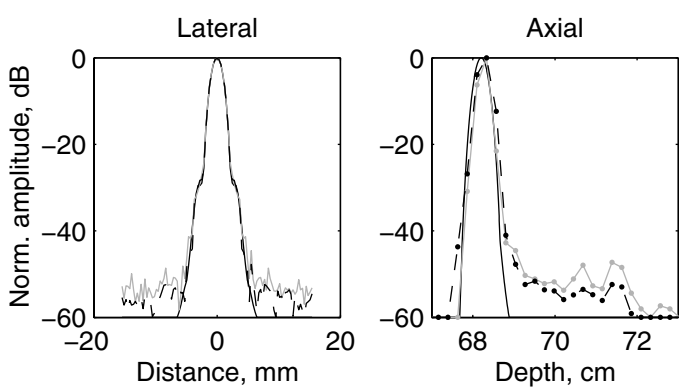

Figure 5: Simulated PSF: classic beamforming (solid line), ideal matched filter (dashed line), shortened filter (grey line).

3. The output data from the DSM is a 1-bit wide in the current implementation and the apodization does not require multiplications. It uses one register instead.

4. The sum operation across all channels is pipelined in order to incorporate numerous inputs and to process them at high clock frequency. The multiplication operation is pipelined also and works at the modulation clock frequency.

5. A chain of beamformers can be used, each of them receiving partially beamformed sample from a neighbor, summing it with its own partially beamformed sample, and passing it further on.

\section{Phantom data processing results}

A set of element traces sampled at $40 \mathrm{MHz}$ was obtained using the experimental sampling system RASMUS [8]. That data was resampled at $140 \mathrm{MHz}$ and $200 \mathrm{MHz}$, and was beamformed according to the suggested architecture. A comparison between the beamforming approaches is shown in Fig. 6. The element number is 32 and the F-number is between 2.5 and 10. It can be seen that the quantization noise of the DSM limits the picture contrast and increasing the OSR improves that. Improvement can also be achieved by employing a more sophisticated modulator architecture.

\section{Conclusion}

A novel flexible beamformer architecture utilizing DSM is suggested. The beamformer can be housed in one standard FPGA, which can easily be programmed and upgraded. Combined with a simple analog front end, the whole design can be implemented by three chips (one of them containing the transmit amplifiers). A standard portable PC can be used for display, making it a very inexpensive system. 

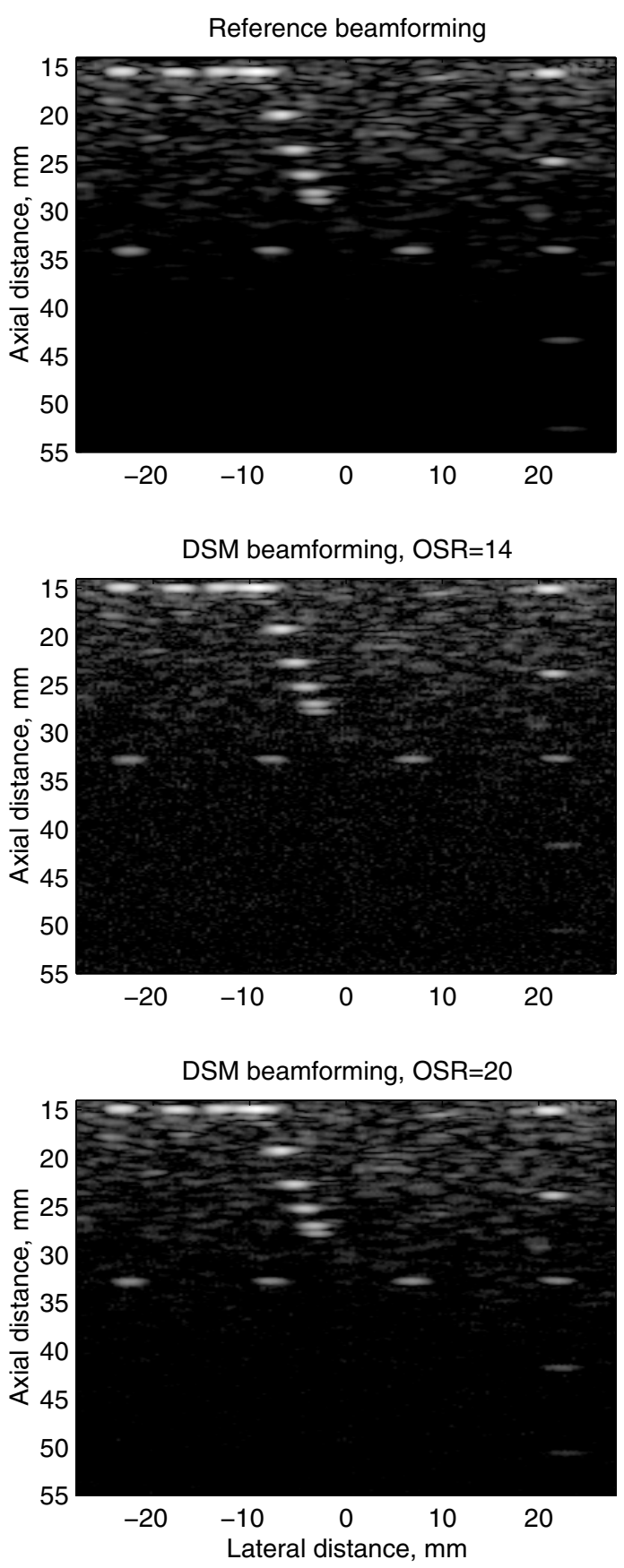

Figure 6: Images created with classic beamforming and with the suggested beamforming at different OSR. The dynamic range is $40 \mathrm{~dB}$

\section{Acknowledgment}

This work was supported by grant 9700883 and 9700563 from the Danish Science Foundation, by B-K Medical A/S, Gentofte, Denmark, by the Thomas B. Thrige Center for Microinstruments, and by the Danish Research Academy.

The authors would like to thank all colleagues at the Center for Fast Ultrasound Imaging for their cooperation and valuable comments on the paper.

\section{References}

[1] J. C. Candy and G. C. Temes. Oversampling Delta-Sigma Data Converters - Theory, Design and Simulation. IEEE Press, 1992.

[2] S. R. Freeman, M. K. Quick, M. A. Morin, R. C. Anderson, C. S. Desilets, T. E. Linnenbrink, and M. O'Donnell. Delta-sigma oversampled ultrasound beamformer with dynamic delays. IEEE Trans. Ultrason., Ferroelec., Freq. Contr., 46:320-332, 1999.

[3] S. R. Freeman, M. K. Quick, M. A. Morin, R. C. Anderson, C. S. Desilets, T. E. Linnenbrink, and M. O’Donnell. Heterodyning technique to improve performance of delta-sigma-based beamformers. IEEE Trans. Ultrason., Ferroelec., Freq. Contr., 46:771-790, 1999.

[4] M. Kozak and M. Karaman. Digital phased array beamforming using single-bit delta-sigma conversion with non-uniform oversampling. IEEE Trans. Ultrason., Ferroelec., Freq. Contr., 48:922-931, 2001.

[5] J. E. Bresenham. Algorithm for computer control of a digital plotter. IBM Systems J., 4:25-30, 1965.

[6] M. Karaman, A. Atalar, and H. Köymen. VLSI circuits for adaptive digital beamforming in ultrasound imaging. IEEE Trans. Med. Imag., 12:711-720, 1993.

[7] H. T. Feldkämper, R. Schwann, V. Gierenz, and T. G. Noll. Low power delay calculation for digital beamforming in handheld ultrasound systems. Proc. IEEE Ultrason. Symp., 2:1763-1766, 2000.

[8] J. A. Jensen, O. Holm, L. J. Jensen, H. Bendsen, H. M. Pedersen, K. Salomonsen, J. Hansen, and S. Nikolov. Experimental ultrasound system for real-time synthetic imaging. In Proc. IEEE Ultrason. Symp., volume 2, pages 1595-1599, 1999. 